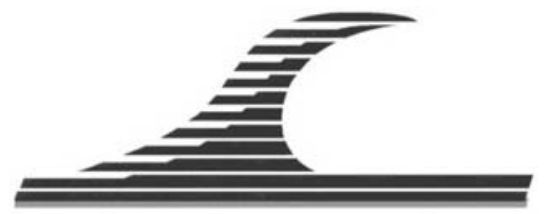

Revue Paralia, Volume 6 (2013) pp 7.1-7.16

Mots-clés : Vagues, Vagues non-linéaires, Vagues dispersives, Houle côtière, Modèle de vagues, Modèle de type Boussinesq,

Technique double-couche.

(C) Editions Paralia CFL

\title{
Validation expérimentale d'un modèle double-couche pour des vagues côtières non-linéaires et fortement dispersives
}

\author{
Michel BENOIT ${ }^{1}$, Florent CHAZEL ${ }^{2}$
}

1. Laboratoire d'Hydraulique Saint-Venant, Université Paris-Est (unité de recherche commune EDF R\&D, CETMEF, Ecole des Ponts ParisTech), 6 quai Watier, BP 49, F-78401 Chatou cedex, France.

michel.benoit@saint-venant-lab.fr

2. Université de Toulouse, Institut National des Sciences Appliquées, Institut de Mathématiques de Toulouse, CNRS UMR 5219, 135 avenue de Rangueil, F-31077 Toulouse cedex 4, France.

florent.chazel@insa-toulouse.fr

\section{Résumé :}

Le modèle original proposé par les auteurs (CHAZEL et al., 2009) pour les vagues en zone côtière est mis en œuvre et validé sur deux cas expérimentaux. Nous considérons d'abord les expériences de DINGEMANS (1994), étudiant la propagation de vagues régulières au-dessus d'une barre trapézoïdale immergée. Les résultats numériques démontrent une excellente capacité du modèle à reproduire les effets de levée, les interactions fortement non-linéaires, ainsi que la génération puis la propagation de composantes harmoniques d'ordres élevés après la barre. Ensuite, nous vérifions la capacité du modèle à propager des vagues irrégulières non-déferlantes sur une bathymétrie plus complexe (essai 26 de BECQ-GIRARD et al., 1999). L'analyse et la comparaison des spectres de variance montrent que les résultats du modèle sont également en très bon accord avec les mesures expérimentales.

\begin{abstract}
The new model recently proposed by the authors (CHAZEL et al., 2009) to simulate waves in the coastal zone is applied and validated on two experimental cases. Firstly the set of experiments by DINGEMANS (1994) is considered, with the propagation of regular waves over a submerged trapezoidal bar. The numerical results show the excellent capabilities of the model to reproduce the shoaling effects, as well as the generation and the further propagation of higher harmonics after the bar. Then we check the ability of the model to propagate irregular non-breaking waves over a more complex bottom profile (experiment 26 by BECQ-GIRARD et al., 1999). The analysis and comparison of variance spectra show that the model results are also in very good agreement with experimental measurements.
\end{abstract}

Keywords: Waves, Nonlinear waves, Dispersive waves, Coastal waves, Wave models, Boussinesq-type model, Two-layer modelling technique.

Article issu d'une sélection des XIèmes Journées Nationales Génie Côtier Génie Civil qui se sont tenues aux Sables d'Olonne du 22 au 25 juin 2010. Accepté le 17 février 2011, en ligne le 28 octobre 2013.

Pour citer cet article :

BENOIT M., CHAZEL F. (2013). Validation expérimentale d'un modèle double-couche pour des vagues côtières non-linéaires et fortement dispersives. Revue Paralia, Vol. 6, pp 7.1-7.16.

DOI:10.5150/revue-paralia.2013.007

(disponible en ligne - http://www.paralia.fr - available online) 


\section{Introduction}

Durant les deux dernières décennies, les modèles de type Boussinesq (plus ou moins étendus) se sont imposés comme une approche de modélisation performante et couramment utilisée par la communauté scientifique et technique pour la modélisation de la dynamique des vagues en zone côtière. A l'origine, les modèles de ce type se basaient sur deux hypothèses fondamentales, celle de faible non-linéarité des ondes de surface et celle de faible dispersion, ce qui leur conférait un domaine de validité limité aux eaux peu profondes et aux vagues de faibles cambrures (voir par exemple PEREGRINE, 1967). De nombreux efforts ont depuis porté sur l'extension du domaine de validité des modèles de ce type, en incluant des termes d'ordres supérieurs pour les effets dispersifs et non-linéaires. En particulier, les travaux de NWOGU (1993) et WEI et al. (1995) tout d'abord, puis ceux de GOBBI et al. (2000), AGNON et al. (1999) et MADSEN et al. (2002) notamment, ont permis de s'affranchir de l'hypothèse de faible non-linéarité, puis de celle de faible dispersion, permettant ainsi de simuler correctement des ondes fortement non-linéaires jusqu'en eaux profondes (typiquement jusqu'à des profondeurs d'eau relatives $k h=6$, où $k$ est le nombre d'onde et $h$ la hauteur d'eau), mais au prix d'un accroissement conséquent de la complexité numérique des modèles développés, liée notamment à l'utilisation de dérivées spatiales d'ordres élevés (i.e. ordres supérieurs à 3 ).

Guidés par la volonté d'obtenir un modèle applicable sur des domaines côtiers de géométries complexes (bathymétrie et trait de côte irréguliers), et précis jusqu'en eaux profondes, mais avec une complexité numérique moindre que les modèles mentionnés ci-dessus, les auteurs ont récemment proposé un nouveau modèle de type Boussinesq fondé sur une approche double-couche (CHAZEL et al., 2009). Ce modèle est présenté dans la partie 2 de cet article, et sa mise en œuvre numérique est exposée en partie 3. Nous illustrons dans la partie 4 les capacités du modèle sur deux cas de validation : les expériences en canal à vagues de DINGEMANS (1994) avec un haut-fond, et un essai de BECQ-GIRARD et al. (1999) réalisé également en canal en conditions de vagues irrégulières sur un profil bathymétrique irrégulier.

\section{Méthodologie de construction du modèle de CHAZEL et al. (2009)}

On considère ici l'évolution d'un fluide à surface libre, non visqueux et incompressible, sous la seule influence de la gravité. On suppose par ailleurs que l'écoulement est irrotationnel, que les effets capillaires liés à la tension de surface peuvent être négligés, et enfin que la pression à la surface est constante, égale à la pression atmosphérique.

Le domaine occupé par le fluide est compris entre un fond (rigide et imperméable) d'équation $z=\bar{Z}(X)=-h(X)$, où $X=(x, y)$ est la variable d'espace horizontale et $z$ la variable verticale, et une surface libre dynamique d'équation $z=\eta(t, X)$. On considère donc que l'élévation de surface libre $\eta$ est une fonction monovaluée de $X$ (i.e. pas de 
retournement de la crête pour des vagues déferlantes). On effectue en outre l'hypothèse de pente douce du fond marin, autrement dit les variations de la bathymétrie sont supposées lentes $(|\nabla h|<<1)$. Enfin, le niveau $z=0$ correspond à la surface du fluide au repos.

\subsection{Formulation double-couche du problème}

La construction du modèle élaboré par CHAZEL et al. (2009) combine trois idées, présentées ci-après dans leurs grandes lignes. La première d'entre elles consiste à utiliser une approche double-couche, telle que proposée par LYNETT \& LIU (2004), où l'on divise le domaine fluide en deux couches, possédant la même masse volumique. Ces couches sont séparées par une interface située à la cote $z=\hat{z}(X)=-\sigma h(X)$, où $\sigma$ est un paramètre constant à choisir dans l'intervalle $] 0 ; 1[$ (voir figure 1 pour une définition du domaine de calcul). Cette séparation étant purement artificielle, on supposera naturellement que les différentes inconnues du problème, telles que le potentiel des vitesses $\phi(t, X, z)$, la vitesse verticale $w(t, X, z)$ et la pression $P(t, X, z)$, sont continues à l'interface entre les deux couches. Ainsi que spécifié par LYNETT \& LIU (2004), le principal but de cette approche est de parvenir à réduire l'ordre des dérivées en espace apparaissant dans le modèle final.

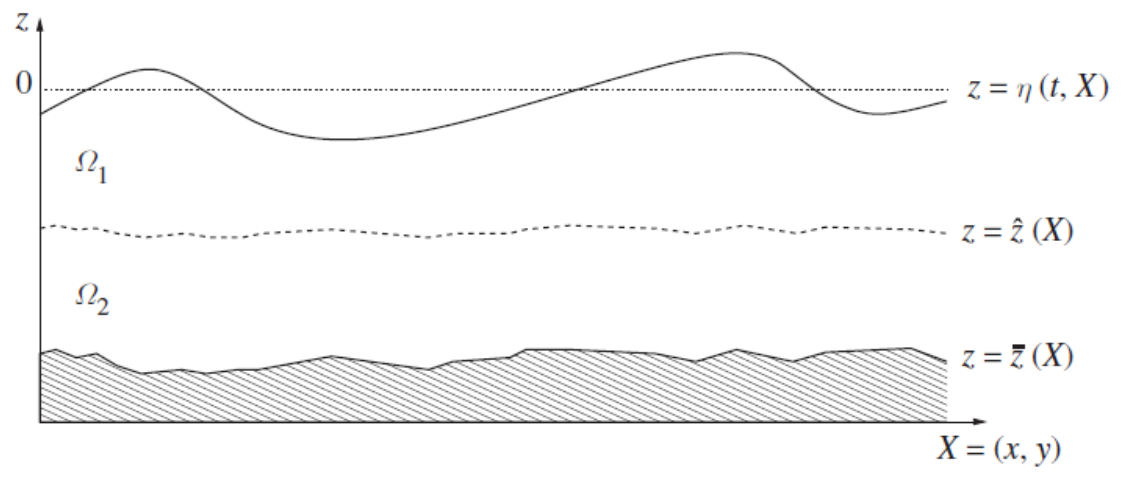

Figure 1. Représentation du domaine fluide et notations principales.

En s'appuyant sur l'hypothèse d'irrotationnalité de l'écoulement, les équations d'Euler à surface libre caractérisant le problème initial peuvent ainsi s'écrire sous forme potentielle, diminuant par là le nombre total d'inconnues par rapport à une formulation en vitesse. Cette formulation est complétée par les conditions de continuité à l'interface entre les deux couches mentionnées précédemment.

Par la suite, en suivant les travaux de ZAKHAROV (1968), on projette les équations précédentes sur la surface libre, afin de travailler sur une formulation 2DH (deux dimensions d'espace horizontales) du problème. Ces équations s'écrivent ainsi : 


$$
\begin{aligned}
& \frac{\partial \widetilde{\phi}_{1}}{\partial t}+\frac{1}{2}\left|\nabla \widetilde{\phi}_{1}\right|^{2}-\frac{1}{2} \widetilde{w}_{1}^{2}\left(1+|\nabla \eta|^{2}\right)+g \eta=0 \\
& \frac{\partial \eta}{\partial t}+\nabla \eta \cdot \nabla \widetilde{\phi}_{1}-\widetilde{w}_{1}\left(1+|\nabla \eta|^{2}\right)=0 \\
& \widetilde{w}_{1}=G[\eta, h] \widetilde{\phi}_{1}
\end{aligned}
$$

où $\widetilde{\phi}_{1}$ et $\widetilde{w}_{1}$ correspondent respectivement au potentiel des vitesses et à la vitesse verticale du fluide à la surface libre $z=\eta(t, X)$, et $G[\eta, h]$ représente l'opérateur de Dirichlet-Neumann, permettant de faire le lien entre les deux quantités précédentes. Pour une fonction $\psi(X)$ donnée, correspondant au potentiel à la surface libre, cet opérateur calcule tout d'abord les potentiels des vitesses $\phi_{1}(X, z)$ et $\phi_{2}(X, z)$ de chaque couche, comme les solutions du problème elliptique aux limites composé :

i) des deux équations de Laplace (une dans chaque couche du fluide),

ii) de la condition de Dirichlet à la surface libre $\phi_{1}(X, \eta)=\psi(X)$,

iii)des conditions de continuité à l'interface entre les deux couches,

iv)de la condition de glissement au fond.

Au final, l'opérateur $G[\eta, h]$ fournit l'évaluation de la vitesse verticale à la surface $\widetilde{w}_{1}$ à partir de la solution $\tilde{\phi}_{1}$ précédente.

Ainsi, la construction du modèle final reposera essentiellement sur la recherche d'une approximation de cet opérateur de Dirichlet-Neumann.

\subsection{Un opérateur de Dirichlet-Neumann statique}

La deuxième idée principale dans le construction du modèle consiste à reformuler les équations précédentes en fonction d'un opérateur de Dirichlet-Neumann alternatif, exprimé non pas à la surface libre dynamique $z=\eta(t, X)$, mais à la surface au repos (i.e. en $z=0$ ). Le principal intérêt de cette reformulation est de passer d'un opérateur dynamique $G[\eta, h]$ à un opérateur statique $G[0, h]$, noté $G_{0}[h]$. Ce dernier permet de faire le lien entre le potentiel $\phi_{0}$ au niveau de la surface libre au repos (i.e. en $z=0$ ), et la vitesse verticale $w_{0}$ au même niveau. La contrepartie à cette approche réside en l'introduction des deux nouvelles inconnues précédentes $\phi_{0}$ et $\mathrm{w}_{0}$, et donc la nécessité de fermer le problème en rajoutant deux nouvelles équations.

Ces équations de fermeture doivent permettre de faire le lien entre les inconnues $\widetilde{\phi}_{1}$ et $\widetilde{w}_{1}$ à la surface libre (i.e. en $z=\eta(t, X)$ ), et les inconnues $\phi_{0}$ et $w_{0}$ au niveau de la surface libre au repos (i.e. en $z=0$ ). Elles peuvent être facilement obtenues en écrivant les développements de Taylor du potentiel $\widetilde{\phi}_{1}$ et de la vitesse verticale $\widetilde{w}_{1}$ entre les cotes $z=0$ et $z=\eta$, combinés avec l'équation de Laplace dans la première couche du fluide. Bien entendu, ces développements doivent être tronqués à un certain ordre, et une brève analyse d'ordres de grandeur montre que l'on obtient une approximation très satisfaisante en tronquant ceux-ci à l'ordre 3. On obtient ainsi la formulation intermédiaire suivante du modèle : 


$$
\left.\begin{array}{l}
\frac{\partial \widetilde{\phi}_{1}}{\partial t}+\frac{1}{2}\left|\nabla \widetilde{\phi}_{1}\right|^{2}-\frac{1}{2} \widetilde{w}_{1}^{2}\left(1+|\nabla \eta|^{2}\right)+g \eta=0 \\
\frac{\partial \eta}{\partial t}+\nabla \eta \cdot \nabla \widetilde{\phi}_{1}-\widetilde{w}_{1}\left(1+|\nabla \eta|^{2}\right)=0 \\
\left(1-\frac{\eta^{2}}{2} \Delta+\left(\eta-\frac{\eta^{3}}{6} \Delta\right) G_{0}[h]\right) \phi_{0}=\widetilde{\phi}_{1} \\
\widetilde{w}_{1}=\left(-\eta \Delta+\left(1-\frac{\eta^{2}}{2} \Delta\right) G_{0}[h]\right) \phi_{0}
\end{array}\right\}
$$

La dernière partie dans la dérivation du modèle réside désormais dans la construction d'une approximation, notée $G_{0}^{\text {app }}[h]$, de l'opérateur de Dirichlet-Neumann statique $G_{0}[h]$.

\subsection{Construction de l'opérateur statique approché $G_{0}^{\text {app }}[h]$}

La dernière idée dans la construction du modèle consiste à adapter à notre formulation double-couche du problème la méthodologie proposée par AGNON et al. (1999) et MADSEN et al. (2002). Dans un premier temps, on recherche dans chaque couche des solutions de l'équation de Laplace sous la forme de séries de Taylor infinies. Les séries obtenues sont ensuite tronquées à un certain ordre, le choix de cet ordre étant motivé par une analyse d'ordres de grandeur.

C'est à ce stade qu'apparaît l'intérêt majeur de notre formulation double-couche. En effet, celle-ci nous permet de choisir un ordre de troncature moins élevé que celui choisi par MADSEN et al. (2002), entraînant ainsi une diminution intéressante (d'un point de vue numérique) de l'ordre des dérivées en espace. Par la suite, des approximants de Padé sont introduits dans les séries tronquées, afin de diminuer plus avant l'ordre maximum des dérivées présentes. Les approximations obtenues des solutions des équations de Laplace dans chaque couche sont ensuite évaluées successivement à la surface libre, à l'interface entre les deux couches, et au fond, afin de traduire les différentes conditions aux limites du problème elliptique lié à l'opérateur de DirichletNeumann statique $G_{0}[h]$.

Après les développements mathématiques détaillés dans CHAZEL et al. (2009), et nonrepris ici, le modèle final s'écrit : 


$$
\left.\begin{array}{l}
\frac{\partial \widetilde{\phi}_{1}}{\partial t}+\frac{1}{2}\left|\nabla \widetilde{\phi}_{1}\right|^{2}-\frac{1}{2} \widetilde{w}_{1}^{2}\left(1+|\nabla \eta|^{2}\right)+g \eta=0 \\
\frac{\partial \eta}{\partial t}+\nabla \eta \cdot \nabla \widetilde{\phi}_{1}-\widetilde{w}_{1}\left(1+|\nabla \eta|^{2}\right)=0 \\
\left(1-\frac{\eta^{2}}{2} \Delta+\left(\eta-\frac{\eta^{3}}{6} \Delta\right) G_{0}^{\text {app }}[h]\right) \phi_{0}=\widetilde{\phi}_{1} \\
\widetilde{w}_{1}=\left(-\eta \Delta+\left(1-\frac{\eta^{2}}{2} \Delta\right) G_{0}^{\text {app }}[h]\right) \phi_{0}
\end{array}\right\}
$$

où l'expression de $G_{0}^{\text {app }}[h]$ est donnée par :

$$
G_{0}^{\mathrm{app}}[h]=Q_{0}\left(\begin{array}{llll}
N_{1} & N_{2} & 0 & 0
\end{array}\right)\left(\begin{array}{cccc}
M_{11} & M_{12} & 0 & 0 \\
M_{21} & M_{22} & M_{23} & M_{24} \\
M_{31} & M_{32} & M_{33} & M_{34} \\
0 & M_{42} & M_{43} & M_{44}
\end{array}\right)\left(\begin{array}{c}
P_{0} \\
Q_{1} \\
Q_{2} \\
Q_{3}
\end{array}\right)
$$

Les différents opérateurs apparaissant dans cette expression (i.e. $N_{\mathrm{i}}, P_{0}, Q_{\mathrm{i}}, M_{\mathrm{ij}}$ ) sont d'ordre deux au maximum (cf. CHAZEL et al. (2009) pour plus de détails). Le modèle final est ainsi composé de quatre équations, que le domaine spatial soit unidimensionnel (1DH) ou bidimensionnel (2DH) dans le plan horizontal, et il n'inclut que des dérivées spatiales d'ordre deux au maximum, ce qui représente un avantage conséquent vis-à-vis des modèles de Boussinesq précédemment cités.

Le principe général de résolution du modèle est le suivant: les deux premières équations du système (3) permettent de faire évoluer en temps le potentiel de vitesse à la surface libre $\widetilde{\phi}_{1}$ et l'élévation de la surface libre $\eta$ respectivement, qui sont les deux inconnues principales du problème. Ces deux équations ne font intervenir que les deux quantités en question ( $\eta$ et $\widetilde{\phi}_{1}$ ), ainsi que la vitesse verticale $\widetilde{w}_{1}$ en surface. Pour estimer cette vitesse, on utilise d'abord la troisième équation de (3) pour déterminer le potentiel $\phi_{0}($ en $z=0)$ à partir de $\widetilde{\phi}_{1}$ et $\eta$ (connus), puis la quatrième équation pour obtenir $\widetilde{w}_{1}$ à partir de $\phi_{0}$.

\subsection{Analyse linéaire du modèle}

Une analyse linéaire du modèle a été menée par CHAZEL et al. (2009) afin d'évaluer les propriétés dispersives du modèle dans sa version linéarisée, en particulier la vitesse de phase, les profils verticaux de vitesse horizontale ainsi que le coefficient de levée (shoaling). Ces propriétés sont comparées aux résultats issus de la théorie linéaire de Stokes. A ce stade, le paramètre $\sigma$, permettant de positionner l'interface entre les deux couches (cf. figure 1), joue un rôle déterminant. En effet, en faisant varier celui-ci, il est possible d'optimiser les propriétés dispersives du modèle (ici dans sa version linéarisée) en minimisant les erreurs commises vis-à-vis de la théorie linéaire de Stokes. Dans 
CHAZEL et al. (2009), il a ainsi été montré qu'en profondeur intermédiaire ( $k h=\pi / 2$ ou $h / L=1 / 4$ ), la valeur $\sigma=0,473$ est optimale, tandis qu'en eau profonde ( $k h=\pi$ ou $h / L=1 / 2$ ), c'est la valeur $\sigma=0,314$ qui est optimale. C'est cette dernière valeur qui a été adoptée sur tous les cas-tests présentés ultérieurement. Néanmoins, plusieurs valeurs de $\sigma$ dans l'intervalle $[0,2 ; 0,5]$ ont été testées, et aucune différence significative n'a été observée dans les résultats pour les cas-tests réalisés par CHAZEL et al. (2009). Ceci démontre une certaine robustesse du modèle vis-à-vis du paramètre $\sigma$, qui n'a a priori d'influence sur les résultats que pour les cas-tests les plus dispersifs.

Les propriétés dispersives du modèle s'avèrent au final excellentes bien au-delà de la limite habituellement considérée pour la grande profondeur d'eau $(k h=\pi)$. A titre d'exemple, les résultats pour la célérité de phase $C=\omega / k=L / T$ pour une onde périodique sur fond plat sont présentés sur la figure 2 , en considérant cette valeur de $\sigma$ pour le modèle. On voit sur cette figure qu'il faut aller jusqu'à une profondeur relative $k h=25$ (environ 8 fois la limite de grande profondeur d'eau) pour atteindre une erreur de $1 \%$ sur la célérité de phase linéaire. Au final, si on se fixe un niveau maximal d'erreur relative de 2\% par rapport aux résultats de la théorie linéaire de Stokes, la version linéarisée du modèle est valable jusqu'aux profondeurs relatives suivantes (voir détails dans CHAZEL et al., 2009) :

$k h=28 \quad$ pour la célérité de phase,

$k h=10 \quad$ pour le profil vertical de potentiel de vitesse,

$k h=8 \quad$ pour le profil vertical de vitesse orbitale verticale,

$k h=12 \quad$ pour le gradient de coefficient de shoaling.

Ces performances en termes de dispersion sont équivalentes à celles obtenues pour le modèle de MADSEN et al. (2002), pour une complexité mathématique et numérique nettement moindre du présent modèle.

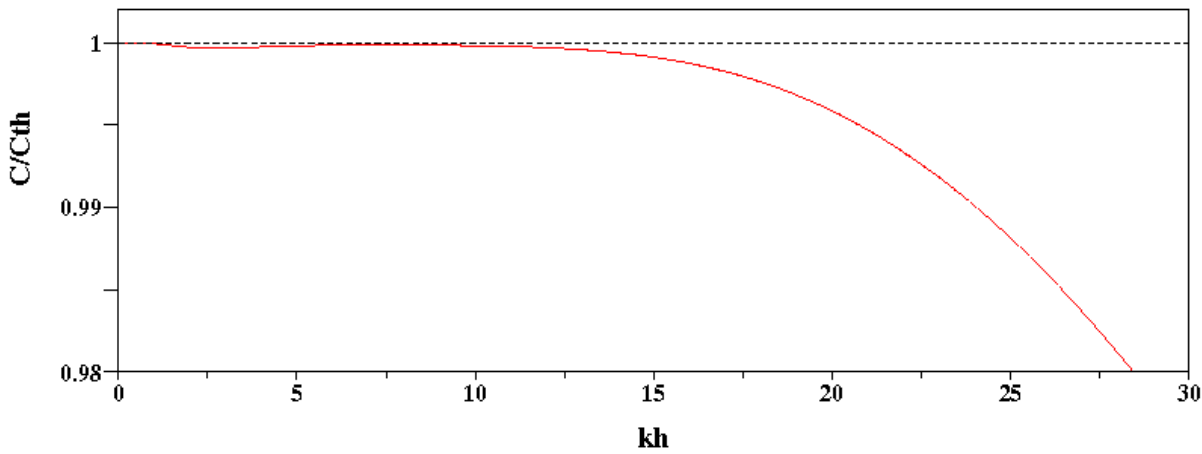

Figure 2. Rapport entre la vitesse de phase du modèle linéarisé (C) et la vitesse de phase de la théorie de Stokes à l'ordre $1\left(C_{t h}\right)$, en fonction de kh (pour $\sigma=0,314$ ) 


\section{Mise en ouvre numérique du modèle en une dimension d'espace horizontale}

Le modèle mathématique développé (3) a été simulé numériquement en une seule dimension d'espace horizontale (modèle 1DH) au travers d'un code de calcul en différences finies développé sous SCILAB ${ }^{\circledR}$ (http://www.scilab.org/). Le domaine de calcul est discrétisé par des mailles de taille constante. Les dérivées premières et secondes sont approchées par des formules centrées d'ordre 4 calculées sur des cellules (stencils) de 5 points consécutifs. Les conditions aux limites sur les inconnues sont imposées de la manière suivante : les coefficients correspondant aux points de la cellule sortant du domaine de calcul sont réfléchis de manière paire pour une condition de type Neumann, et impaire pour une condition de type Dirichlet homogène. Cette méthode permet en particulier de conserver une structure très régulière dans les matrices de discrétisation.

Afin de reproduire numériquement le comportement d'un bassin à vagues, des zones de relaxation sont mises en place en entrée et en sortie du domaine de calcul (cf. BINGHAM \& AGNON, 2005) afin d'assurer la génération et l'absorption des vagues. Sur ces zones de relaxation et à chaque pas de temps, la valeur finale de chaque inconnue est calculée par combinaison convexe de la solution numérique et de la solution que l'on cherche à imposer. Cette dernière pourra par exemple correspondre aux expressions du potentiel et de la surface libre issues du développement de Stokes à l'ordre 1 ou données par une méthode de type "fonction de courant", ou bien être identiquement nulle dans le cas d'une zone d'absorption. Dans les deux cas, des conditions de type Neumann sont imposées en conditions aux limites sur le potentiel et la surface libre au niveau des frontières latérales.

Enfin, l'intégration en temps du modèle est effectuée à l'aide d'un schéma de RungeKutta explicite à 4 pas, d'ordre 4 , avec un pas de temps $\Delta t$ constant, qui possède un domaine de stabilité étendu. Dans certains cas traités par CHAZEL et al. (2009), du fait des non-linéarités en jeu, des instabilités de hautes fréquences ont pu été observées. Dans ce cas, pour y remédier CHAZEL et al. (2009) ont proposé d'appliquer un filtre de Savitzky-Golay d'ordre 8 à la fin de chaque pas de temps sur les variables $\eta, \widetilde{\phi}_{1}$ et $\widetilde{w}_{1}$, et vérifié que l'usage de ce filtre a un effet négligeable sur la précision des résultats en sortie. Cependant, pour les simulations des deux cas expérimentaux présentés dans la partie suivante, aucune instabilité n'a été observée et il n'est donc pas fait usage d'un quelconque filtrage ou lissage des résultats au cours des simulations.

\section{Validation du modèle sur deux cas expérimentaux}

\subsection{Propagation de vagues régulières au-dessus d'une barre immergée}

Nous examinons ici la capacité du modèle à représenter la propagation et les interactions non-linéaires entre des ondes relativement dispersives. Pour cela, nous nous 
intéressons à la propagation de vagues régulières au-dessus d'une barre trapézoïdale immergée, en suivant le dispositif utilisé par DINGEMANS (1994) et décrit sur la figure 3. Des vagues régulières sont générées en entrée de domaine (frontière gauche) et se propagent en direction de la barre. Au passage de celle-ci, la diminution de la profondeur entraîne un raidissement du front des vagues, ainsi qu'une augmentation de la hauteur des vagues. Les interactions vague-vague non-linéaires génèrent des composantes harmoniques d'ordres élevés, qui se propagent de façon liée au mode fondamental et donnent des profils de vagues asymétriques (par rapport à un plan horizontal et un plan vertical). Ces composantes harmoniques sont ensuite libérées sur la partie plate du profil, puis lorsque la profondeur d'eau augmente à nouveau, pour devenir des ondes fortement dispersives se propageant librement au-delà de la barre. Une partie significative de l'énergie des vagues se situe alors dans le domaine $k h>4$, ce qui rend ce cas-test très exigeant au niveau des propriétés dispersives et non-linéaires du modèle testé.

On présente tout d'abord les résultats du cas A $(H=2,0 \mathrm{~cm} ; T=2,02 \mathrm{~s})$ pour lequel les ondes incidentes sont relativement longues $(k h=0,67$ au large de la barre) et de cambrure relativement faible $(k H / 2=0,017$ ou $H / L=0,0053)$.

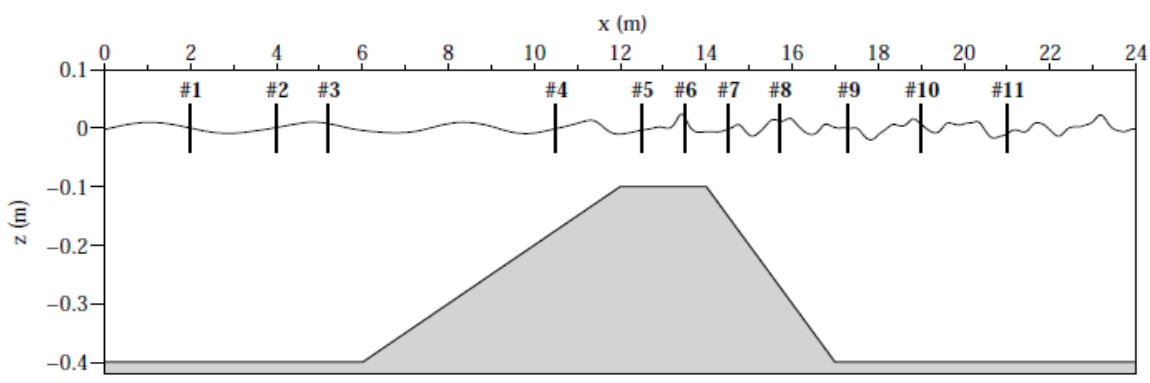

Figure 3. Dispositif expérimental de DINGEMANS (1994) : profil bathymétrique et positions des sondes de mesure de vagues.

Sur la figure 4, les profils de surface libre simulés pour le cas A sont comparés aux mesures expérimentales sur une durée de 2 périodes de vague incidente sur les 4 dernières sondes numérotées 8 à 11 sur la figure 3. Ces sondes, situées derrière la barre, sont celles en lesquelles les effets dispersifs se manifestent de la façon la plus marquée. On distingue en effet nettement l'effet de "désintégration" du train d'onde incident par la barre avec la présence d'harmoniques d'ordres supérieurs, qui se propagent avec leur propre célérité (au contrainte de la partie située avant le replat où les harmoniques restent liées au mode fondamental). Cela se traduit par des profils temporels de surface libre très différents d'une sonde à l'autre en fonction des phases relatives des composantes harmoniques ainsi libérées. 

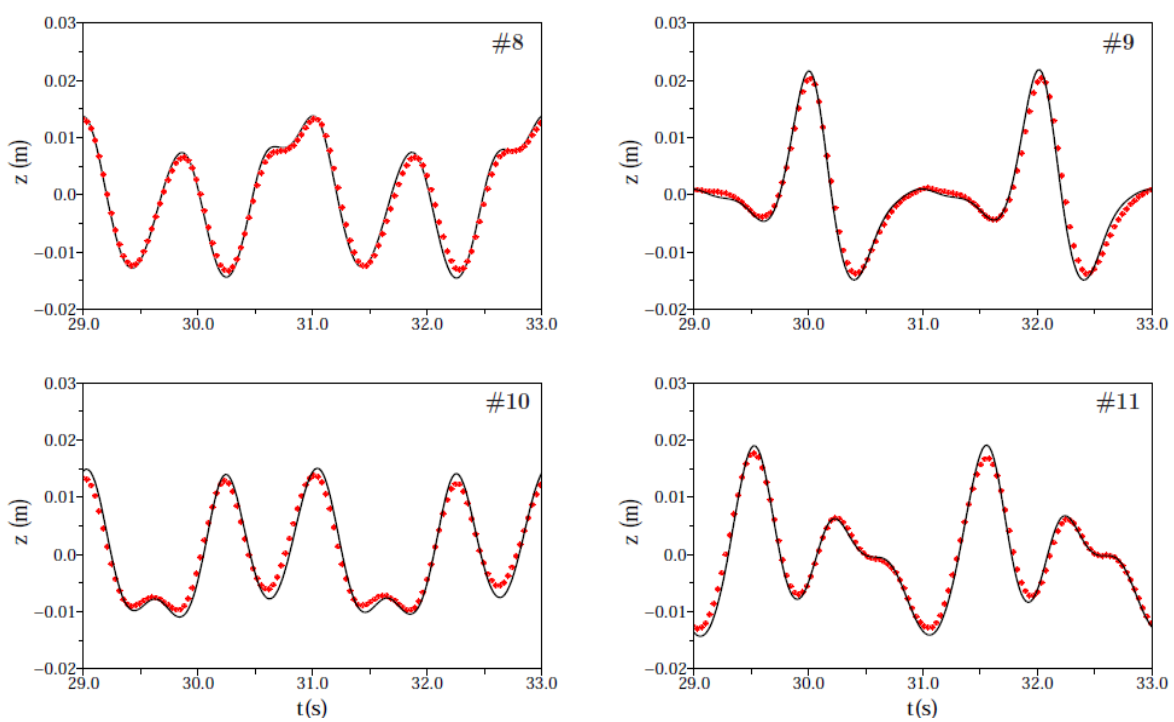

Figure 4. Profils de la surface libre aux dernières sondes pour le cas $A(H=2,0 \mathrm{~cm}$; $T=2,02 \mathrm{~s})$ : résultats du modèle en trait continu, résultats expérimentaux en points.
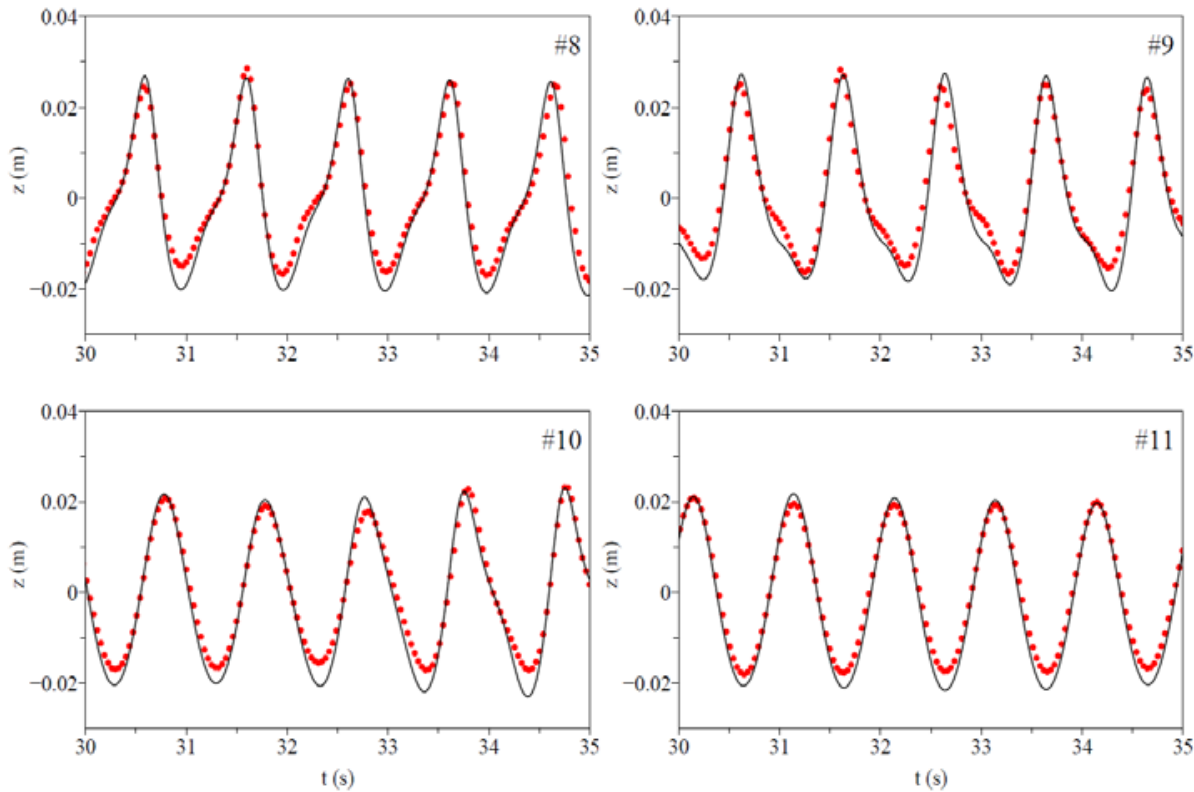

Figure 5. Profils de la surface libre aux dernières sondes pour le cas $C(H=4,1 \mathrm{~cm}$; $T=1,01 \mathrm{~s})$ : résultats du modèle en trait continu, résultats expérimentaux en points.

Nous avons également simulé le cas $\mathrm{C}$ de cette même série d'expériences, caractérisé par une période de vague incidente plus courte de moitié par rapport au cas A $(T=1,01 \mathrm{~s})$ et une hauteur de vague plus importante $(H=4,1 \mathrm{~cm})$. Pour ce cas où les vagues incidentes sont plus courtes $(k h=1,69$ au large de la barre) et présentent une cambrure plus importante $(k H / 2=0,087$ ou $H / L=0,028)$, les résultats du modèle sont également très bons, comme illustré sur la figure 5 pour les profils de surface libre aux quatre dernières sondes de mesure. Sur ce cas C, la part d'énergie transférée du mode 
fondamental vers les composantes harmoniques supérieures est cependant plus faible (en relatif) que sur le cas A, ce qui se traduit par un champ de vagues moins irrégulier après le passage de la barre.

Dans les deux cas simulés, les profils de surface libre sont très bien reproduits, pour toutes les sondes situées derrière la barre, que ce soit en terme d'amplitude ou de phase, y compris pour les détails des harmoniques 2 et 3 . L'accord des résultats obtenu ici est bien meilleur que celui que l'on peut attendre de modèles de type Boussinesq étendu tels que ceux de NWOGU (1993) ou WEI et al. (1995) par exemple.

\subsection{Propagation de vagues sur des fonds complexes en faible profondeur d'eau}

Une série d'essais en vagues irrégulières a été réalisée en 1998 à EDF R\&D Chatou dans le cadre de la thèse de F. Becq-Girard (voir BECQ-GIRARD et al., 1999) pour un profil bathymétrique, représenté sur la figure 6 , destiné à mettre en évidence les effets non-linéaires en faible profondeur d'eau. Le canal considéré (canal $n^{\circ} 12$ ) a une longueur de $45 \mathrm{~m}$ pour une largeur de $0,60 \mathrm{~m}$. Il est équipé d'un batteur plan, animé d'un mouvement de type "piston" et piloté par ordinateur. Le profil bathymétrique étudié se compose d'une succession de pentes (plaques métalliques lisses) dont l'inclinaison diminue jusqu'à l'arrivée sur un replat; puis la profondeur augmente à nouveau légèrement, avant de diminuer régulièrement sous la forme d'une pente de plage constante (pente d'environ 5\%). Les séries temporelles d'élévation de surface libre étaient mesurées en 16 points répartis le long du profil, comme indiqué sur la figure 6 , à l'aide de sondes résistives.

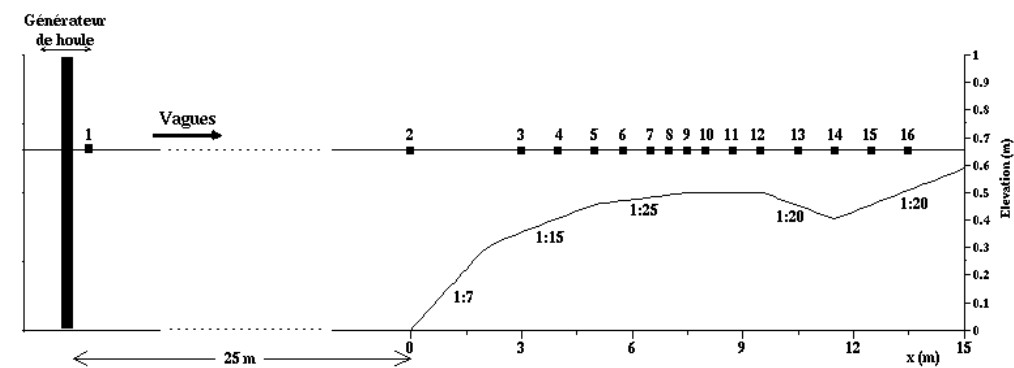

Figure 6. Dispositif expérimental des essais en canal de BECQ-GIRARD et al. (1999) : profil bathymétrique et positions des sondes de mesure de vagues.

Nous considérons ici l'essai 26 de cette série d'expériences, qui correspond à un essai en vagues irrégulières et sans déferlement. La profondeur d'eau au large du profil bathymétrique et au niveau du générateur de vagues est $h=0,65 \mathrm{~m}$. La hauteur significative des vagues générées est de $H_{m o}=3,4 \mathrm{~cm}$ pour une fréquence de pic de $f_{p}=0,435 \mathrm{~Hz}$ (période de pic $T_{p}=2,3 \mathrm{~s}$ ). Le spectre de vague imposé en consigne au générateur de vagues est de type JONSWAP, avec un facteur d'élancement du pic $\gamma=3,3$. Les mesures ont été acquises sur une durée de $40 \mathrm{~min}$, correspondant à un peu 
plus de 1000 vagues de période de pic $T_{p}$, avec un pas de temps d'échantillonnage de $0,070 \mathrm{~s}$ (soit environ 34 points par vague de période de pic $T_{p}$ ). Le spectre mesuré dans le canal à la sonde 2 (située en début du profil bathymétrique) est représenté sur la figure 7 (en échelle logarithmique pour la densité de variance).

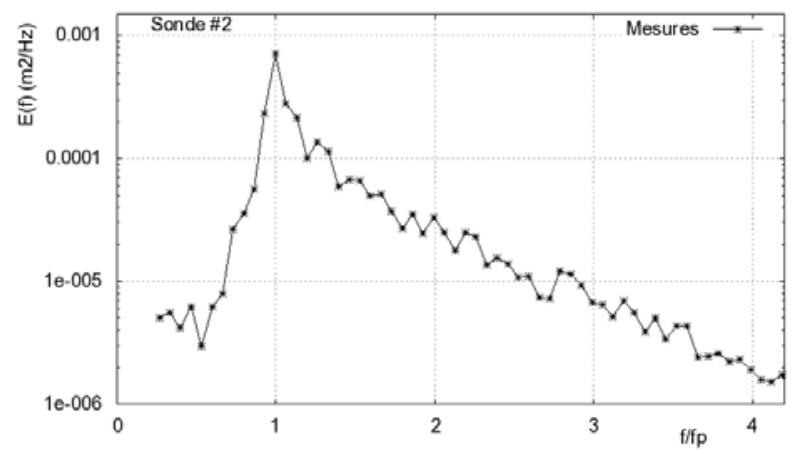

Figure 7. Spectre de variance de surface libre mesuré à la sonde 2 (en début de profil bathymétrique) pour l'essai 26 de BECQ-GIRARD et al. (1999).

Le maillage de calcul utilisé sur ce cas couvre une distance de $25 \mathrm{~m}$ de $x=-5 \mathrm{~m}$ à $x=+20 \mathrm{~m}$, avec une taille de maille $\Delta x=0,10 \mathrm{~m}$; il comprend donc 250 mailles en espace. Cette résolution spatiale correspond à environ 65 points par vagues de période de pic $T_{p}$, pour la profondeur d'eau $h=0,65 \mathrm{~m}$ au large du profil bathymétrique. Le pas de temps utilisé est de $\Delta t=0,0657 \mathrm{~s}\left(=T_{p} / 35\right)$ et la simulation couvre une durée de $1000 T_{p}=2390 \mathrm{~s} \approx 40 \mathrm{~min}$. La série temporelle d'élévation de surface libre mesurée à la sonde 2 est utilisée comme condition à la limite de la simulation, de sorte que les spectres de variance mesurés et simulés sont identiques pour cette sonde.

Les profils temporels de surface libre obtenus avec le modèle ont été comparés aux mesures expérimentales aux différentes sondes, avec un très bon accord général, comme l'illustre par exemple la figure 8. Cette figure présente un extrait des signaux temporels mesurés et simulés au niveau de la dernière sonde (sonde 16) sur une durée de $15 \mathrm{~s}$ (soit une durée d'à peu près 6,5 périodes de pic). Le signal réel, pourtant très irrégulier après sa propagation sur tout le profil bathymétrique, est très bien reproduit par le modèle numérique. Les crêtes de vagues (et les creux) sont bien modélisés, tout comme les légères oscillations du profil liées aux composantes harmoniques d'ordres élevés. Ces composantes harmoniques semblent se propager avec les vitesses de phase correctes, ce qui confirme la capacité du modèle à reproduire tant les interactions non-linéaires que la propagation d'ondes fortement dispersives. 


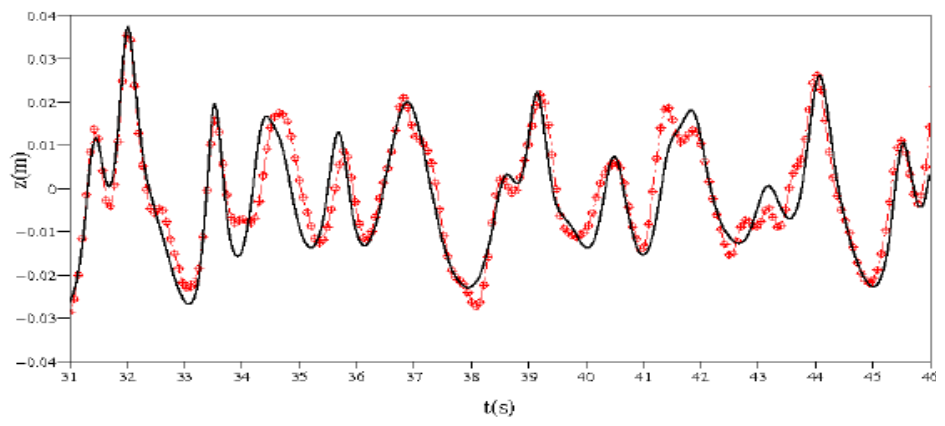

Figure 8. Profil de surface libre à la sonde 16 pour le cas 26 de BECQ-GIRARD et al. (1999) : résultats du modèle en trait continu, résultats expérimentaux en trait pointillé avec symboles.

Une analyse plus détaillée est effectuée sur la base des spectres de variance aux différentes sondes. Les signaux d'élévation de surface libre mesurés et simulés numériquement sont traités par un programme d'analyse spectrale basé sur la méthode du périodogramme utilisant les mêmes options dans les deux cas. Les résultats sont présentés sur les figures 9 (spectres aux sondes 4, 5, 7 et 9) et 10 (spectres aux sondes $11,13,15$ et 16). Sur ces graphes, la fréquence, en échelle linéaire, est normalisée par la fréquence de pic du spectre (de façon à mieux identifier les pics harmoniques aux fréquences $2 f_{\mathrm{p}}, 3 f_{\mathrm{p}}$ et $4 f_{\mathrm{p}}$. Pour la densité de variance (axe vertical), une échelle logarithmique a été utilisée, de façon à mieux distinguer les niveaux d'énergie portés par les différentes fréquences harmoniques.

L'évolution des spectres aux différentes sondes permet de bien mettre en évidence les mécanismes physiques en jeu. Dans un premier temps, lorsque la profondeur d'eau diminue sur la pente externe du profil, on note d'une part une amplification de la hauteur du pic principal (sonde 4) du fait du shoaling, et d'autre part un transfert d'énergie progressif vers les pics harmoniques à $2 f_{\mathrm{p}}$ (sondes 4 et 5 ), $3 f_{\mathrm{p}}$ (sonde 7 ), et même $4 f_{\mathrm{p}}$ (sondes 9 et 11). Le développement de ces pics harmoniques résulte des effets non-linéaires dans la propagation des vagues à mesure que la profondeur d'eau diminue ; leurs amplitudes sont très bien restituées par le modèle numérique, y compris pour la composante harmonique à $4 f_{\mathrm{p}}$. Après le haut-fond, lorsque la profondeur d'eau augmente, on note une diminution de l'amplitude des pics harmoniques de plus hautes fréquences : d'abord sur la sonde 13, puis sur la sonde 15 pour laquelle seuls le pic principal et l'harmonique à $2 f_{\mathrm{p}}$ portent un niveau d'énergie significatif (et avec des amplitudes de pic de même ordre de grandeur). Enfin, au niveau de la sonde 16 lorsque la profondeur d'eau diminue à nouveau, l'amplitude du pic harmonique à $3 f_{\mathrm{p}}$ augmente à nouveau, du fait de la reprise des effets non-linéaires. Là encore, cette tendance est très précisément restituée par le modèle numérique. 

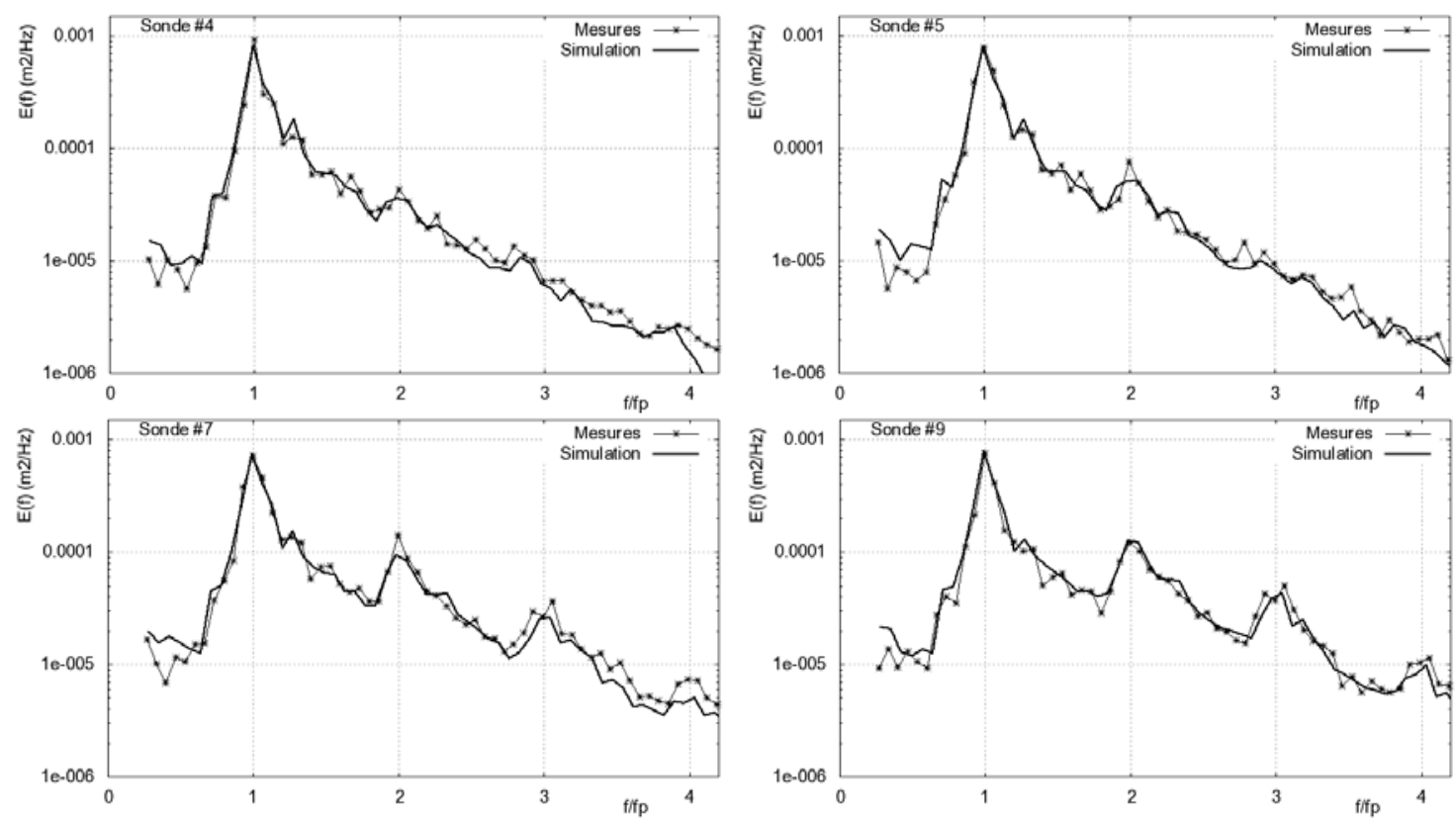

Figure 9. Spectres de variance de surface libre mesurés et simulés pour l'essai 26 de BECQ-GIRARD et al. (1999) : sondes 4, 5, 7 et 9 (voir positions sur la figure 6).
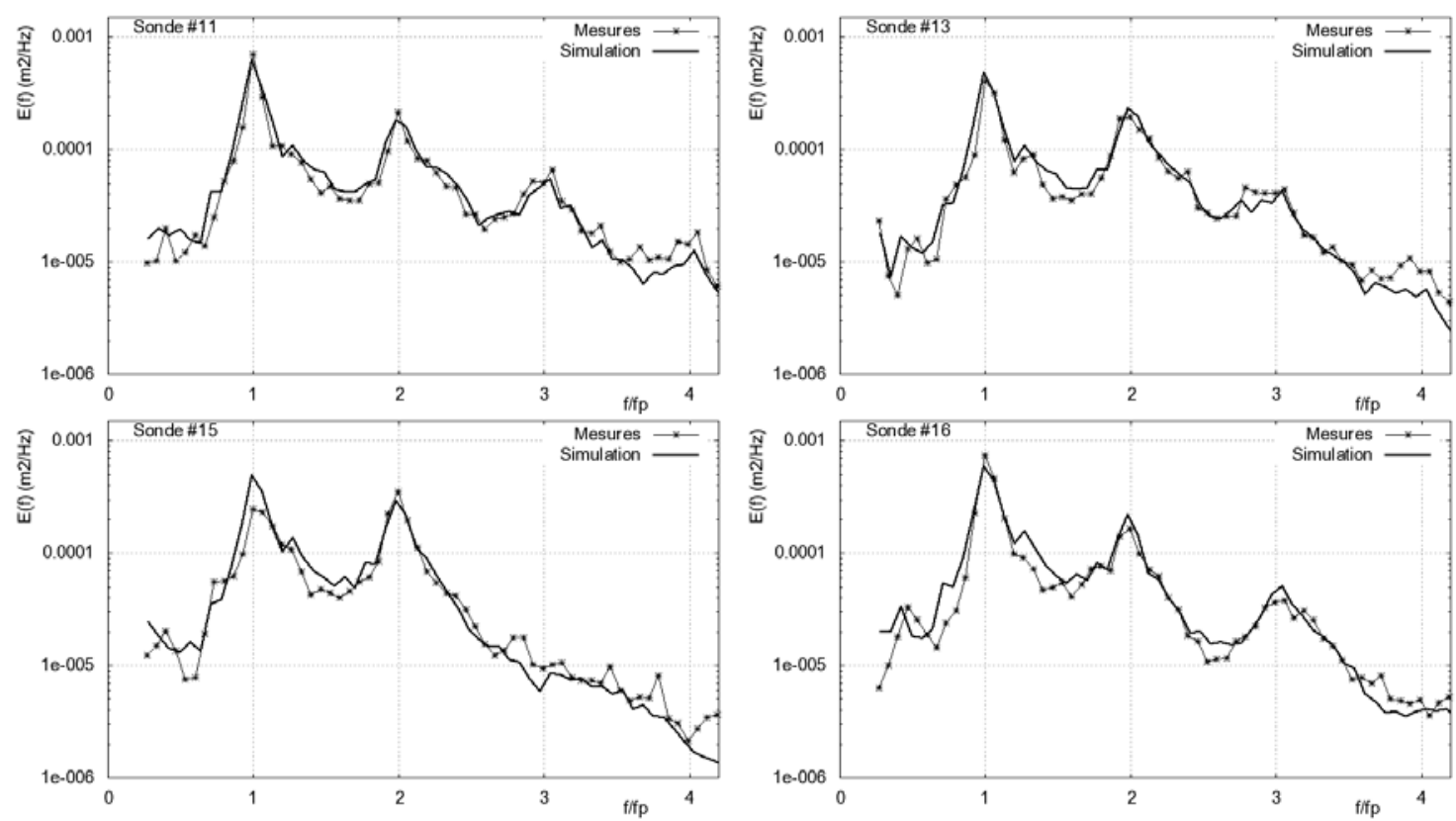

Figure 10. Spectres de variance de surface libre mesurés et simulés pour l'essai 26 de BECQ-GIRARD et al. (1999) : sondes 11, 13, 15 et 16 (voir positions sur la figure 6).

\section{Conclusions et perspectives de développement}

Le modèle mathématique (3) proposé par CHAZEL et al. (2009) pour la modélisation des vagues en zone côtière possède plusieurs avantages. D'un point de vue mathématique et numérique, il n'est composé que de quatre équations que l'on traite des 
Validation expérimentale d'un modèle double-couche pour des vagues côtières non-linéaires et fortement dispersives : 7.15

cas en une ou deux dimensions d'espace horizontales; il ne comporte que des dérivées spatiales d'ordre deux au maximum et il concentre l'essentiel de sa complexité dans un opérateur Dirichlet-Neumann approché qui est statique (i.e. qui peut être calculé une fois pour toutes en début de simulation). Il possède de plus des propriétés linéaires dispersives extrêmement étendues, jusqu'en eaux profondes (bien au-delà de la limite de grande profondeur $k h=\pi$ ).

Les deux validations expérimentales présentées et analysées dans cet article (expériences en canal à vagues de DINGEMANS (1994) et de BECQ-GIRARD et al. (1999)) confirment les très bonnes capacités du modèle au niveau dispersif, mais également au niveau de la modélisation des effets d'interactions non-linéaires. Dans les deux cas, les transferts non-linéaires sont correctement modélisés et les effets dispersifs très bien restitués, y compris pour des profils bathymétriques non-monotones (avec des hauts-fonds) et pour des états de mer irréguliers. Les résultats obtenus sur ces cas-tests exigeants sont de très bonne facture, et supérieurs à ceux de modèles de type Boussinesq étendu tels que NWOGU (1993) ou WEI et al. (1995) par exemple. Ils permettent d'anticiper un bon comportement du modèle sur des cas réels avec bathymétrie irrégulière et emprise du domaine de calcul s'étendant jusqu'à des profondeurs relatives importantes (supérieures à $k h=10$ ).

Les travaux actuels concernent la réalisation de cas-tests de validation complémentaires, avant d'étendre ensuite le modèle aux cas de situations 2DH avec l'objectif d'utiliser des maillages non-structurés. Au plan physique, on envisage ultérieurement d'ajouter la prise en compte de processus dissipatifs d'énergie, déferlement et cisaillement au fond notamment.

\section{Références Bibliographiques}

AGNON Y., MADSEN P.A., SCHÄFFER H.A. (1999). A new approach to high-order Boussinesq models. Journal of Fluid Mechanics, Vol. 399, pp 319-333. doi:10.1017/S0022112099006394

BECQ-GIRARD F., FORGET P., BENOIT M. (1999). Non-linear propagation of unidirectional wave fields over varying topography. Coastal Engineering, Vol. 38, pp 91-113. doi:10.1016/S0378-3839(99)00043-5

BINGHAM H.B., AGNON Y. (2005). A Fourier-Boussinesq method for nonlinear water waves. European Journal of Mechanics B/Fluids, Vol. 24, pp 255-274. doi:10.1016/j.euromechflu.2004.06.006

CHAZEL F., BENOIT M., ERN A., PIPERNO S. (2009). A double-layer Boussinesqtype model for highly nonlinear and dispersive waves. Proceedings of Royal Society of London, Series A, Vol. A 465, pp 2319-2346. doi:10.1098/rspa.2008.0508

DINGEMANS M. (1994). Comparison of computations with Boussinesq-like models and laboratory measurements. Mast-G8M note, H1684, Delft Hydraulics, Pays-Bas. 
GOBBI M.F., KIRBY J.T., WEI G. (2000). A fully nonlinear Boussinesq model for surface waves. Part 2. Extension to $\mathrm{O}\left((\mathrm{kh})^{4}\right)$. Journal of Fluid Mechanics, Vol. 405, pp 181-210. doi:10.1017/S0022112099007247

LYNETT P., LIU P.L.-F. (2004). A two-layer approach to wave modelling. Proceedings of Royal Society of London Series A, Vol. A 460, pp 2637-2669. doi:10.1098/rspa.2004.1305 MADSEN P.A., BINGHAM H.B., LIU H. (2002). A new Boussinesq method for fully nonlinear waves from shallow to deep water. Journal of Fluid Mechanics, Vol. 462, pp 1-30. doi:10.1017/S0022112002008467

NWOGU O.G. (1993). Alternative form of Boussinesq equations for nearshore wave propagation. Journal of Waterway, Port, Coastal and Ocean Engineering, Vol. 119, $\mathrm{n}^{\circ}$ 6, pp 618-638. doi:10.1061/(ASCE)0733-950X(1993)119:6(618)

PEREGRINE D.H. (1967). Long waves on a beach. Journal of Fluid Mechanics, Vol. 27, pp 815-827. doi:10.1017/S0022112067002605

WEI G., KIRBY J.T., GRILLI S.T., SUBRAMANYA R. (1995). A fully nonlinear Boussinesq model for surface waves: Part I. Highly nonlinear unsteady waves. Journal of Fluid Mechanics, Vol. 294, pp 71-92. doi:10.1017/S0022112095002813

ZAKHAROV V.E. (1968). Stability of periodic waves of finite amplitude on the surface of a deep fluid. Journal of Applied Mechanics and Technical Physics, Vol. 9, pp 190-194. doi:10.1007/BF00913182 